tion of release is produced by adding oxotremorine, a stimulant of cholinergic muscarinic receptors. The inhibition of acetylcholine release in either case is abolished by the cholinergic muscarinic receptor antagonist drug atropine, which by itself does not alter acetylcholine release. The authors suggest that acetylcholine may act to inhibit its own release by way of muscarinic receptors, and that several previous reports that atropine can cause a stimulation of acetylcholine release can probably be explained by the fact that cholinesterase inhibitors were used in these experiments. In such conditions the high concentration of acetylcholine accumulating in the extracellular space may have caused an artefactual inhibition of transmitter release.

There have been many reports that the rate of release or turnover of the monoamine transmitters noradrenaline, dopamine and 5-hydroxytryptamine in the mammalian central nervous system is generally lower in response to the administration of receptor stimulating drugs and increases in response to receptor blocking drugs. Such findings have been described by the generic term "receptor feedback", and have generally been interpreted in terms of complex polysynaptic reflex pathways. The present findings suggest that some of these phenomena may bear re-examination in the light of the possible existence of local "auto-inhibition" or "prostaglandin" feedback controls at the various different types of presynaptic terminal.-From our Neuropharmacology Correspondent.

\section{Gumma-ray Statistics}

ONE needs a certain grim determination to be a gammaray astronomer these days. It is now fourteen years since experimentalists were fired with enthusiasm by a paper by Morrison (Nuovo Cim., 7, $858 ; 1958$ ) which stressed the importance of making astronomical observations in the gamma-ray region of the spectrum; but, in spite of the expenditure of a great deal of money and effort, there have been very few discoveries in gamma-ray astronomy.

The potential importance of this branch of astronomy is easy to understand. Observations in radio astronomy have shown that many regions of the Universe contain large fluxes of very energetic particles. The physical conditions in these regions are still poorly understood, but there is little doubt that the energetic particles should produce gamma-rays in interactions with matter, with radiation and with magnetic fields. Understanding of these regions should, therefore, benefit greatly from observations in the gamma-ray part of the spectrum.

Several basic experimental problems have hindered the development of gamma-ray astronomy. The Earth's atmosphere strongly absorbs all but the highest energy gamma-rays and the measurements must be made from high altitude balloons or from satellites. There is also a large background flux of gamma-rays produced in interactions of cosmic-ray particles with the Earth's atmosphere or with the detector itself. Finally, the flux of gamma-rays which can be expected from even the strongest sources is very small. This means that statistical fluctuations seriously limit the sensitivity of detectors.

The development of the spark chamber in the 1960s gave a fresh impetus to gamma-ray astronomy. At ener- gies greater than $10 \mathrm{MeV}$ gamma-rays interact with a detector through the process of pair production, and the direction of the electron-positron pair - and hence the direction of the gamma-ray-can be measured accurately in a spark chamber. During the 1960 s, larger and larger spark chambers were built for experiments on high altitude balloons ; the biggest now has an area of $2,500 \mathrm{~cm}^{2}$ and the balloons provide exposure times of the order of a day. In 1972 the SAS-B satellite, containing a spark chamber with a collecting area of $225 \mathrm{~cm}^{2}$, was launched.

An alternative experimental approach is possible for gamma-rays with energies greater than $10^{6} \mathrm{MeV}$. When a gamma-ray of this energy interacts with the Earth's atmosphere it creates an electromagnetic cascade of electrons, positrons and gamma-rays. The electrons and positrons produce Cerenkov radiation in the atmosphere, and this reaches the ground as a pool of light several hundred metres in diameter. This light can be detected, and its direction can be measured by means of a photomultiplier at a focus of a concave mirror. The effective area of this detecting system is the area of the pool of Cerenkov light, which is very much larger than the area of the mirror, and this helps to compensate for the very low number of gamma-rays which can be expected at these energies. This technique has been fully exploited by the group at the Smithsonian Observatory, led by Fazio and Weekes, which has constructed a concave reflecting system $10 \mathrm{~m}$ in diameter at Mt Hopkins, Arizona.

The results of all this experimental effort have been disappointing. It was soon realized that the early theoretical predictions were far too optimistic; in fact, gammaray astronomers still do not know how sensitive their detectors should be if they are to develop this branch of astronomy. Of course, the past fourteen years have not been uneventful. Whenever any band of faithful devotees search doggedly for the truth, some will see hallucinations. In gamma-ray astronomy hallucinations take the form of statistical analyses, carelessly applied. One may not agree with Ehrenfest, who is reputed to have told a student, "If you need to use statistics to support your argument, then you are probably wrong anyway" ; but one seems to read too often of results which "... have a very low probability of being due to a chance fluctuation" but, eventually, turn out to be just that.

On page 376 of this issue of Nature O'Mongain attempts to apply rigorous statistical tests to data published in recent experiments. He concludes that, at an energy of approximately $100 \mathrm{MeV}$, four sources have been detected -..-the pulsar in the Crab nebula, two sources in Sagittarius and one in Libra. At energies greater than $10^{6} \mathrm{MeV}$ only the Crab pulsar has been detected. No doubt, some experimentalists will disagree with O'Mongain's analysis ; but few will deny that the subject benefits from an occasional critical survey of this kind.

This year will be a crucial one for gamma-ray astronomy. The SAS-B satellite was launched late in 1972 and, apparently, is functioning well. By the end of the year there may well be many more well authenticated sources. The experimental problems will still be severe because astronomy is concerned not only with detecting sources but also with measuring their size and position, measuring spectra and detecting time variations, all of which will require detectors with even greater sensitivities. But when one has cleared Beecher's first time round, the rest of the course looks much less formidable.-R. R. H. 